\title{
Mixing of solids in different mixing devices
}

\author{
INGRID BAUMAN, DUŠKA ĆURIĆ and MATIJA BOBAN
}

Faculty of Food Technology and Biotechnology, University of Zagreb, Pierottijeva 6, 10000 Zagreb, Croatia

e-mail: ibauman@pbf.hr; dcuric@pbf.hr; matija.boban@pbf.hr

MS received 27 August 2007; revised 22 April 2008

\begin{abstract}
Mixing of powders is a common operation in any industry. Most powders are known to be cohesive, many agglomerate spontaneously when exposed to humid atmosphere or elevated storage temperature. Agitation of the powder (especially powders with different bulk densities) may result in migration of smaller particles downwards and of larger ones upwards. Another problem is segregation whose main cause is the difference in particle size, density shape and resilience. There are standard mixing devices, such as drum tumblers or Turbula mixers. Alternate device type used is the static mixer of Kenics type. Static mixers save energy, disable segregation and effect particle migration. In this paper, static mixers, as devices for powder mixing, are tested as well as Turbula and V-shaped drum mixer, since those devices are commonly used for powder blending in industry. Mixtures that were blended by means of those three devices were made out of the model material, quartz sand, in different component ratios (20:80 and 30:70). The results were statistically calculated and graphically presented. Cohesion indexes were measured with Powder Flow Analyser to see the effect of material flow on the mixture quality. The results obtained by those three devices, the particle size effect and cohesion indexes, bring us to the conclusion that static mixers could be used for mixing of powders, but their shape, number of mixing elements and the mixer length should be adapted for each mixture separately, experimentally and mathematically, through modelling of the system.
\end{abstract}

Keywords. Blending; flowability; mixer; parametric statistic.

\section{Introduction}

Mixing of ingredients, or dispersion of one phase into another, is an operation widely used in industry (Lacey 1943). The aim of these operations is to make homogeneous product using the minimum amount of energy and time. Difficulties will appear due to the diversity of products in terms of size - particles or granules, shape-irregularly shaped particles, moisture and surface nature - cohesive or non cohesive powders (Gyebis \& Katai 1990). In blending or mixing of different kinds of particulate matter, one is concerned with three broad aspects. Mixer type selected or designed and the mode of its operation as one, characterization of state of the resultant mixture and the rate of mechanism of the mixing process giving rise 
to this state (Muttio et al 2004). The mixing process is profoundly influenced by the flow characteristics of the particulate matter to be mixed (Mort \& Riman 1995). Recognition of the existence of two types of the particulate matter, free flowing and cohesive, forms the basis for classifying and characterizing mixtures and mixing processes. Differences in the physical properties promote mixing and demixing, and it has become practically impossible to foresee the point of best homogeneity. The mechanism of mixing (Pool et al 1962) is the result of a complex hydrodynamics and rheological properties as well as of chemical and physical interactions between the components. The shape variations in powders are huge and they range from extreme degrees of irregularity to approximately spherical or well-defined crystalline shapes. Both physical and chemical properties of the material, geometry, size and surface characteristics of individual particles and the history of the system as a whole determine the bulk properties of fine powders (Gilbertson \& Eames 2003). Most powders are known to be cohesive. Many powders agglomerate spontaneously when exposed to moist atmosphere or elevated storage temperature. The phenomenon (Gyebis \& Katai 1990) can result in anything between small soft aggregates that break easily, to rock hard lumps of variable size, or solidification of the whole powder mass. Another immense problem in dealing with powders is segregation that occurs when particles of different properties are distributed in different parts of the bed. The main differences responsible for segregation are differences in the particle size, density, shape and resilience (Ottino \& Khakhar 2000), which cannot be avoided in real systems (Railley et al 1994). The segregation process (Ristow 2000) generally occurs when free flowing powders having a significant range of particle size are exposed to some kind of mechanical motion; the particles will migrate to the bottom of the bed so that their concentration decreases as a function of the bed height. Differences in these properties can also cause unmixing or segregation during mixing or mechanical jiggling of the mixture. Generally, materials similar in size, shape, and density are able to form the most uniform mixtures.

Mixing devices (Naranjan et al 1994) can be classified into two groups with respect to segregation: segregating mixers-which have mainly diffusive mechanisms, encouraging the movement of individual particles, making segregation more significant, non-impeller type mixers tend to be of this type.

Less segregating mixers-have mainly convective mixing mechanisms. These are typically impeller types in which blades, screws, ploughs, etc. sweep groups of particles through the mixing zone. Mixing devices are chosen according to the material mixed; therefore, it is important to know the particle size as well as their flow properties. The variability of powders arises from the many ways in which their flow properties may be changed such as: physical properties of the powder particles such as their size, size range, shape, hardness, elasticity, porosity, mass, interactions between particles, texture, angularity and so on. Environmental factors that affect the powder bulk properties, such as the air or moisture content, external pressure, vibration, etc. These factors modify the physical distribution and arrangement of the particles in the powder mass. Individual particle changes caused by factors such as attrition, agglomeration, electrostatic charge and chemical changes.

Taking into account the above mentioned factors, the experiments were done with three different mixing devices. The first one was a classical V-shaped mixer widely used in the industry for mixing powders (Lakshman \& Cooney 2006). The second type was the 'Turbula' mixer (Turbula T2C, 2004) known for giving good results in mixing powders. The third mixing device used was a static mixer, not a typical mixing device for powders, but most frequently used for mixing liquids and non Newtonian fluids. Our interest was to find out how a static mixer reacted while mixing powders and if it could substitute some of the classical mixers 


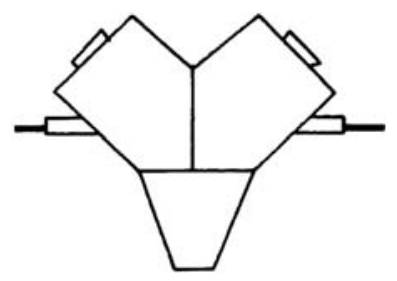

Figure 1. V-shaped drum mixer.

considering material characteristics (particle size, flow properties) especially as an additional device for filling machines to prevent segregation.

\section{Materials and methods}

Three different mixing devices used were: a standard V-shaped rotating drum mixer, Turbula type shaker mixer and a Static mixer. This comparative work was aimed at assessing the impact of varying process parameters such as the particle size, fill level and the rotation speed on the mixing behaviour.

V-blender (figure 1) consists of two cylindrical sections joined at an angle of around $90^{\circ}$, it is designed for batch operation. The angle between the cylinder and the centreline in the off-axis rotating drum mixer is $40^{\circ}$. The mixer is rotated about a horizontal axis, with mixing resulting from the tumbling motion of the particles. The volume fraction of the powders charged in the mixer is between 50 and ' $60 \%$ '. The material falls as the blender turns, ultimately towards the apex and then towards the legs of the ' $\mathrm{V}$ '. The particles of the material move in both vertical and horizontal directions so that a complete mixing occurs. The capacity of the vessel was 2 Ltrs. gross and provided with an opening with a cover for charging and discharging of the product.

The Turbula shaker-mixer (figure 2) is used for a homogeneous mixing of powdery substances with differing specific weights and particle sizes. The product is mixed in its own closed container. The exceptional efficiency of the Turbula shaker-mixer arises from the use of rotation, translation and inversion according to the Schatz geometric theory. The mixing container is set into a three-dimensional movement that exposes the product to continuously changing, rhythmically pulsing motion. It operates by tumbling the solids inside a revolving vessel, at speeds up to about $40 \mathrm{rpm}$ (about half the critical speed at which the centrifugal

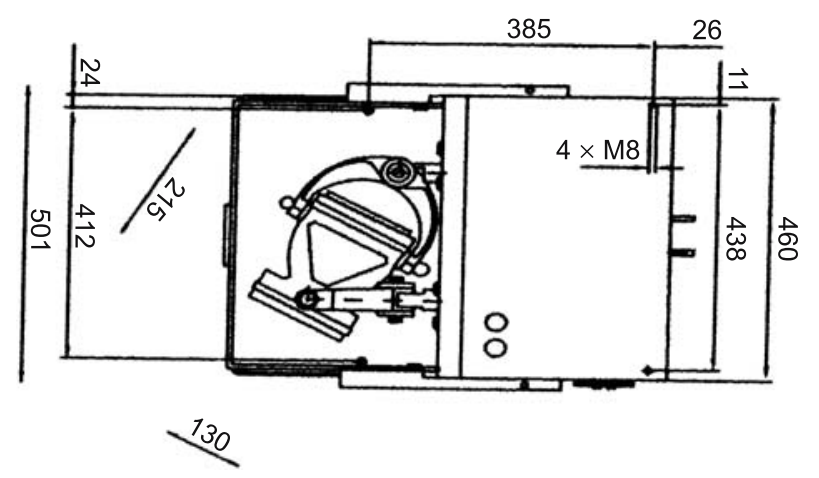

Figure 2. Turbula mixer. 
Table 1. Physical properties of quartz sand.

\begin{tabular}{lccc}
\hline Material & Diameter $(\mathrm{mm})$ & Bulk density $\left(\mathrm{kg} \mathrm{m}^{-3}\right)$ & Cohesion index \\
\hline Quartz sand & $0 \cdot 125$ & $1436 \cdot 70$ & 10 \\
Quartz sand & $0 \cdot 4-0 \cdot 8$ & 1679.90 & 13 \\
\hline
\end{tabular}

force on the particles exceeds the pull of gravity), its working capacity is about $50 \%$ to $60 \%$ of volume. Physical dimensions of the Model 2TF Turbula blender characteristics as well as other mixing devices are given in table 1.

In a static mixer (figure 3) the main mechanism in laminar flow (Reynold's number $<2000$ ) is the flow division. Element styles are three helical or pseudo-helical, and are arranged in a series of alternating left and right hand $180^{\circ}$ twists. The leading edge of an element, which is on a diameter, is at $90^{\circ}$ to the trailing edge of the upstream element. In flow division, the leading edge of the first element splits the material entering the mixer into 2 streams, which are then rotated through $180^{\circ}$. The second element splits the flow again, this time into 4 streams, followed by a further rotation, in the opposite direction, through $180^{\circ}$. The third element repeats the process by splitting into 8 streams, and so on. As the number of streams or layers increases, the layer thickness decreases. Mixture quality is a function only of the mixer diameter and the number of elements and, in the laminar flow, is independent of the flow rate or viscosity. In solid/solid mixing the unit is usually vertical with the flow being by gravity only. The material is removed below the mixer by an assembly line. The mixer must be kept full because whenever there is a free area to form a flow cone or a moving surface, resegregation can occur.

The experiments were carried out with quartz sand which is a non-cohesive free flowing material (table 2) with particles that differed in size, distribution and bulk density.

All the materials were sieved prior to use, so that the components of different granulometric sizes could be obtained. The study was designed to focus on the mixer performances. Mixtures were designed with the components of different particle size, different material ratios, and with
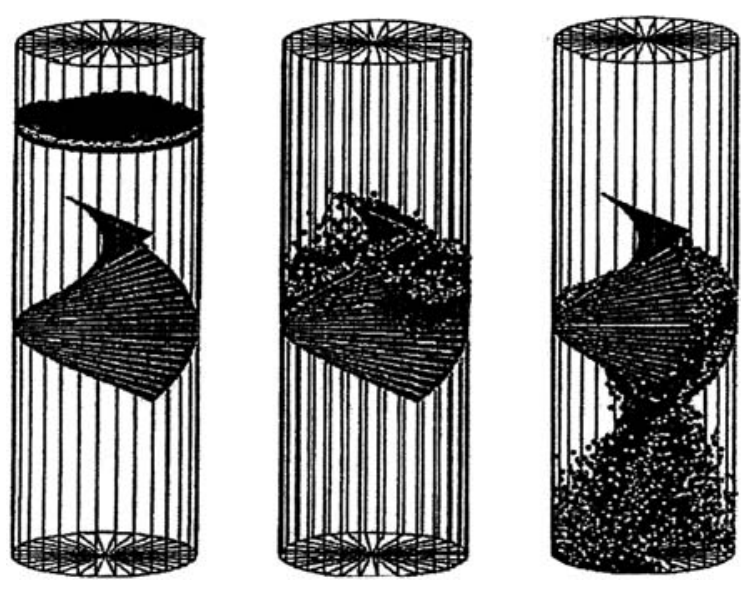

Figure 3. Static mixer. 
Table 2. Mixing devices and their characteristics.

\begin{tabular}{|c|c|c|c|c|c|}
\hline Mixer type & $\begin{array}{c}\text { Tube } \\
\text { length }(\mathrm{m})\end{array}$ & $\begin{array}{c}\text { Tube } \\
\text { width (m) }\end{array}$ & $\begin{array}{l}\text { Number of } \\
\text { elements } \\
\text { in the tube }\end{array}$ & $\begin{array}{l}\text { Height/width } \\
\text { ratio of the } \\
\text { tube }\end{array}$ & $\begin{array}{l}\text { Width of the } \\
\text { element }(m)\end{array}$ \\
\hline Kenics type & $0 \cdot 250$ & $0 \cdot 030$ & $1-5$ & $8 \cdot 3$ & 0.028 \\
\hline Mixer type & $\begin{array}{l}\text { Volume } \\
(\mathrm{ml})\end{array}$ & $\begin{array}{c}\text { Optimal } \\
\text { filling }(\mathrm{ml})\end{array}$ & $\begin{array}{c}\text { Critical no. } \\
\text { of rotation } \\
\left(\mathrm{r} \mathrm{min}^{-1}\right)\end{array}$ & $\begin{array}{c}\text { Drum } \\
\text { length }(\mathrm{mm})\end{array}$ & $\begin{array}{c}\text { Drum } \\
\text { width }(\mathrm{mm})\end{array}$ \\
\hline V-type drum & 830 & 498 & 80 & 120 & 73 \\
\hline Turbula & 2500 & 1500 & 42 & 250 & 110 \\
\hline
\end{tabular}

a large surplus of one of the components. Experiments with the same material combinations were implemented using all three different types of mixing devices.

After the material had been mixed, the samples were analysed. A continuous sampling, paying attention to the main rules of sampling, tested the dynamics of mixing (Bauman 2001). The powder mixed in the static mixer was sampled when in motion and the whole of the stream was taken in 25 second intervals and analysed by two different methods (Muzzio et al 2003). One was a standard granulometric analysis, and the other was done by the 'Sympatec Helos Vectra' laser particle analyser. The data obtained in this way were calculated and treated statistically and the results were presented graphically. Mixing efficiency was measured by the standard deviation of the minor component with respect to the bulk stream at the mixer exit for a static mixer. When mixing in drum mixers (Turbula, V-blender), after a certain amount of time, the mixer was stopped, the content of the cylinder was emptied onto a flat surface and divided into identical parts—-sub-samples. Sub-sample weight was taken and then the sub-samples were sifted through a sieve related to a diameter of used particles. The mass of overflow stream as well as the mass of underflow stream were individually weighed to ascertain the component ratio in the sample. The entire procedure was then repeated within a different interval during which the samples were taken. For analysing the samples in the 'Sympatec Helos Vectra' laser particle analyser, sub-samples were taken in the same way and put into the analysing device. The results showed how many particles of each component were represented in each sample at a given mixing time (figure 4).

The mathematical model was made on the basis of a standard deviation at the beginning and during the mixing process, and on the basis of the interchangeable portion of the components, with the average values of all samples. From standard deviation parameters a simple regression model for mixing dynamics was made (Bauman \& Futač 1996). The model assumed that a variance change curve could be described by adding exponential functions for mixing and demixing. Regression model can be represented with equation:

$$
S^{2}(t)=S_{o}^{2} e^{-k_{1} t}+S_{\infty}^{2}\left(1-e^{-k_{2} t}\right)+S_{a}^{2} .
$$

Values for $S_{o}^{2}$ (variance at the beginning of mixing) are defined from volume content. Measured values of variance modification during the mixing process are used to estimate parameters $S^{2}$ (variance at time $t$ ), $S_{a}^{2}$ (variance of samples containing $n_{a}$ agglomerates), $k_{1}$ and $k_{2}$ (coefficient 


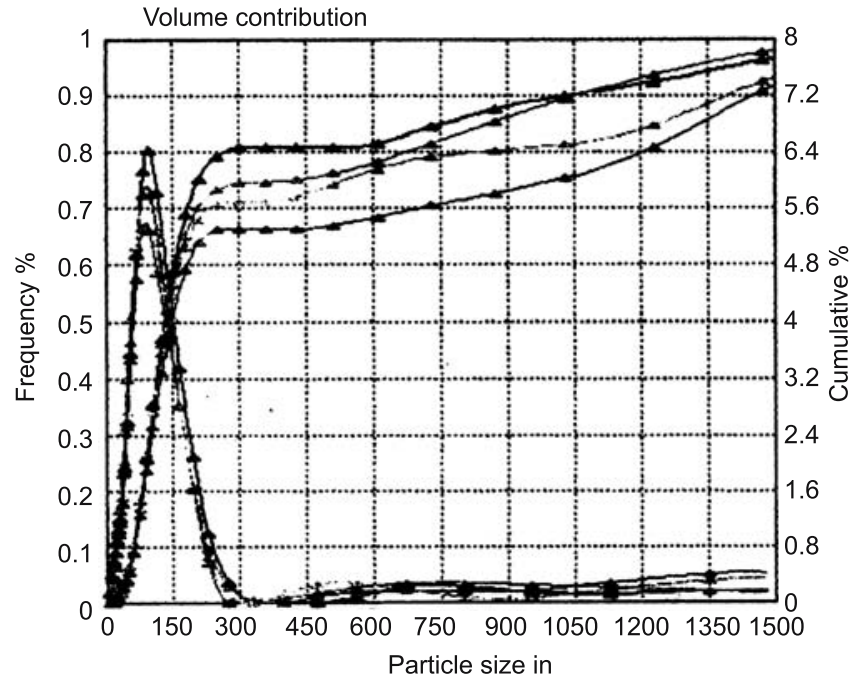

Figure 4. Particle size analysis for quartz sand.

in a model). For calculation nonlinear least squares method was used. As a result of functions nonlinearity iterative procedure was used, and is repeated until values of parameter is reached which can accomplish farther decreasing divergence between measured data and regression model. After the parameters of regression model are estimated the optimal mixing time can be calculated as the minimum of the mixing variance model curve.

$$
\begin{aligned}
& \frac{d S^{2}}{d t} \int_{t_{o p t}}=0 \\
& t_{o p t}=\frac{1}{k_{1}-k_{2} \ln \left(\frac{k_{1} S_{o}^{2}}{k_{2} S_{\infty}^{2}}\right)} .
\end{aligned}
$$

Statistical analysis of the acquired data was conducted by Microsoft Excel program and by the mathematical analysis computer program (Muzzio et al 2003).

Flow properties of the material were tested on the 'Powder Flow Analyser' produced by Stable Micro System (Juliano et al 2006). The instrument was being hailed as an accurate and reliable method of measuring the flow characteristics of dry and wet powders and it could be used to assess any product capable of flow-not just free-flowing powders. Samples were conditioned at the beginning to eliminate any variations in loading, and the precisionengineered, patented blade was then rotated through the sample, causing a 'controlled flow'. Test sequences were repeated ad infinitum to maximize accuracy and repeatability. The samples were placed in a borosilicate glass sample vessel (volume used is ' $160 \mathrm{ml}$ '). During the test, axial force, time and distance were measured by a sensitive transducer and data were displayed and analysed in real time by 16 or 32 bit software. Rheology of the material was measured involving a conditioning, followed by the test cycle during which all the forces acting on the blade were measured. $100 \mathrm{~mm} \mathrm{~s}^{-1}$ speed and 10 degree helix angle blade was used. The result was the basic flowability energy (BFE), expressed in $\mathrm{mJ}$. The basic flowability energy is the energy required to displace a constant volume conditioned powder at a given flow pattern and flow rate. Generally, powders have a flow rate index greater than 1 and 
a high flow rate index signals a 'potentially difficult to process' material since its flow energy and, therefore, its flow behaviour, can change significantly during processing.

\section{Results and discussion}

A mixture can be defined as homogenous if every sample of the mixture has the same composition and properties as any other. The results have been presented through a standard deviation. (Hersey 1982) defined an ordered mixture as having zero standard deviation of the sample concentration at all sample sizes provided that the sample size is greater than the size of a single order unit as opposed to a random mixture where the standard deviation decreases with the increasing of the sample size.

The main problem with mixing powders is their determination in the mixture before and after the mixing process (Kaye 1997). The dynamics of mixing has been tested by continuous sampling. Granulometric analysis explains it only partially; the rest of the problem must be characterized by statistics. The important thing is to get reproducibility, which has been achieved, considering low values of standard deviation obtained in all samples (from 0.05 to $0 \cdot 1$ ). Kenics type of a static mixing device has been investigated, which has been proven as the best type of a powdered material (Regner et al 2006). Static mixers can replace standard device types and they can contribute to the mixtures quality in processes such as backfilling material during packaging. Many producers mix several batches before they start the packaging of the material. During storage of different batches, the powders have a tendency to segregate. A static mixer added to storage devices outlet would lead to the final products quality.

The ratio of the components in the mixture is most important, since it is, after the differentiation of particle size, one of the main reasons for segregation or agglomeration in the blend. It was easy to predict that in 1:1 ratio of different components the homogeneity would be high as shown in figure 5. For static mixer, the model is added to the chart to prove the mixtures quality. The number of elements in the tube is of great importance when static mixers are concerned, because an inadequate number of elements can cause over mixing which leads to a product of unsatisfactory quality. As shown in figure 6 the experiments were conducted with a different number of elements (from 1 to 5) as well as with an empty tube (no elements), to prove that without elements there is no mixing. As for quartz sand, which is

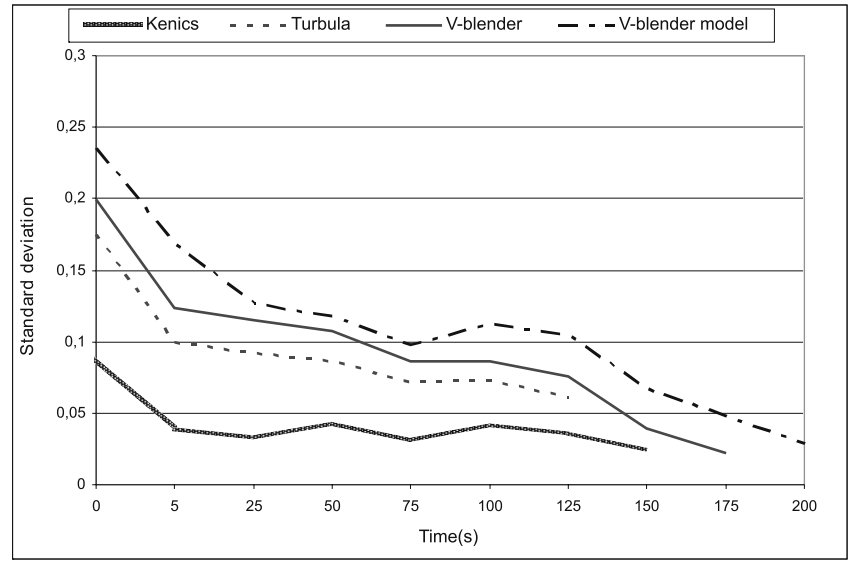

Figure 5. Mixtures with 1:1 ratio of components mixed in different mixing devices together with model sample for static mixer. 

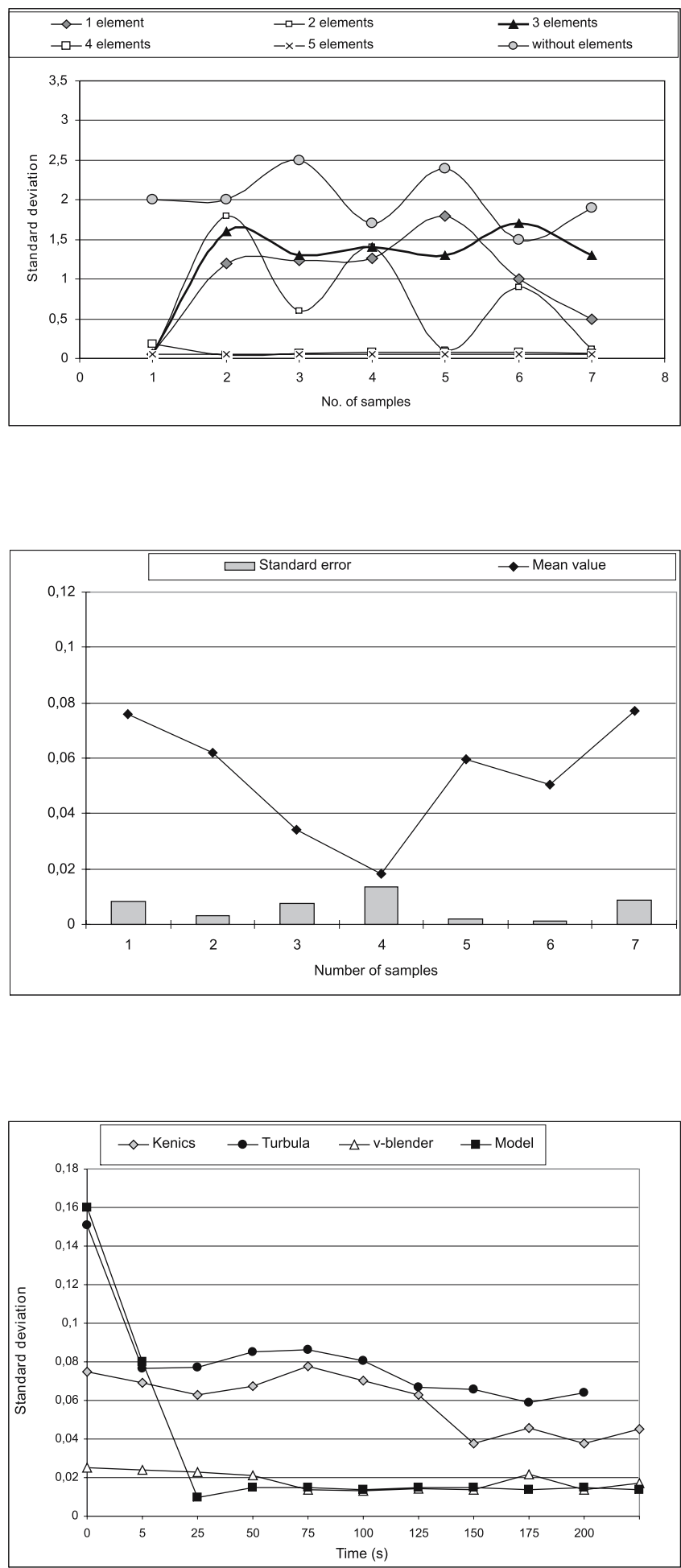

Figure 6. Static mixer with a different number of elements.

Figure 7. Standard error for mean value of mixture in $1: 1$ ratio mixed in a static mixer.

Figure 8. Mixtures with 1:3 ratios (smaller component in surplus) of components mixed in different mixing devices. 


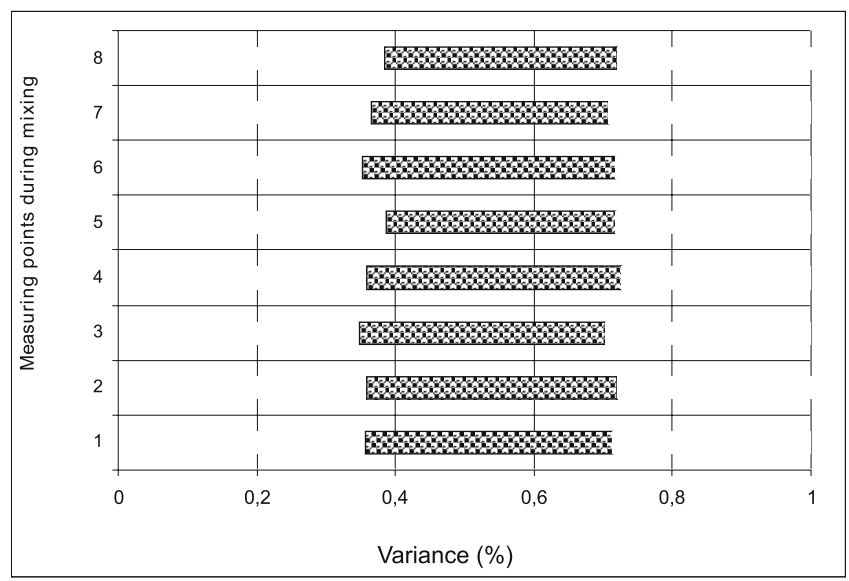

Figure 9. Dissipation of variance values during mixing in a static mixer (1:1 ratio).

model material, 4 to 5 elements gave the best mixture quality, which was additionally proven mathematically, with the model, and presented in figure 6. A standard error for a two component mixture blended in a static mixer is below 0.02 , when components are represented in 1:1 ratio, which is very low as shown in figure 7 and support its claim of a good quality mixture.

When the ratio of components was changed, and one of them is in great surplus (1:3) a much better mixture quality was obtained as shown in figure 8 presented by lower values for standard deviation $(0.01$ to 0.05$)$ which could be explained with packaging of particles between themselves; small particles flowed easier between the large ones which anticipated the mixing rate (Tomas 2004). With the diffusion mechanism, particles migrated through a dilated or expanded powder bed. Figure 9 shows a dissipation of variance value during mixing in a static mixer when component ratio is $1: 1$. The ability of the bed to dilate and the ability of particles to migrate depended highly on the cohesive strength of powder. Powders with little cohesive strength dilate more readily, therefore, shorter blend times can be achieved if the major component of the blend is more free flowing. If convection is considered, a moderately cohesive powder may blend faster because chaotic patterns are introduced (Prescott \& Barnum 2000). A better blend may be achieved if the minor component is somewhat cohesive or has a tendency to adhere to the major component of the blend. Flow rate and cohesion index measured can also add to this conclusion as shown in table 3. Each material by itself

Table 3. Flow behaviour of different particle size quartz sand and its mixtures.

\begin{tabular}{|c|c|c|c|c|}
\hline Sample & $\begin{array}{c}\text { Sample } \\
\text { weight }(\mathrm{g})\end{array}$ & $\begin{array}{l}\text { Cohesion } \\
\text { coefficient } \\
\left(\mathrm{g} \mathrm{mm}^{-1}\right)\end{array}$ & $\begin{array}{l}\text { Cohesion } \\
\text { index }\end{array}$ & $\begin{array}{c}\text { Flow } \\
\text { behaviour }\end{array}$ \\
\hline Quartz sand 1. $(0.125 \mu \mathrm{m})$ & 482.75 & $-2229 \cdot 2$ & $10 \cdot 0$ & Free flowing \\
\hline Quartz sand 2. $(0.4-0.8 \mu \mathrm{m}$ & $499 \cdot 05$ & -3091.68 & $13 \cdot 0$ & Easy flowing \\
\hline Quartz sand mixture (10\% 2:90\% 1) & $501 \cdot 37$ & $-3945 \cdot 24$ & $16 \cdot 0$ & Cohesive \\
\hline Quartz sand mixture (30\% 2:70\% 1) & $483 \cdot 70$ & -3401.65 & $15 \cdot 0$ & Cohesive \\
\hline
\end{tabular}




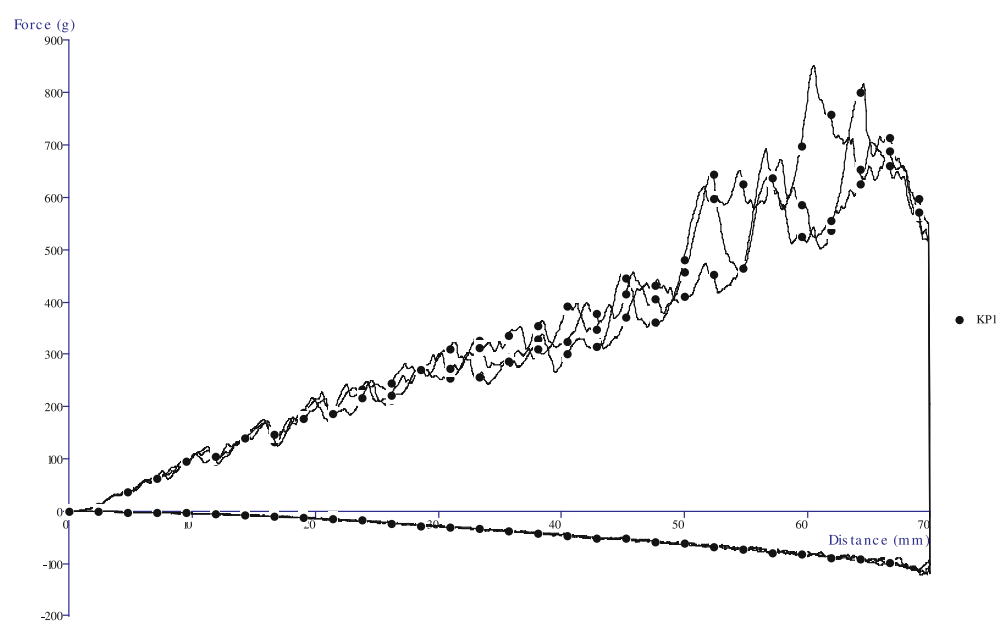

Figure 10. Graph representing measurement of cohesion index for quartz sand. Area of the curve below the abscissa is the force from which coefficient index is calculated.

(quartz sand of different particle size) behaves as free or easy flowing (figure 10). Their flow characteristics change when mixed together and they become cohesive (Janssen 2001). This parameter, if measured before mixing, can help choose mixing parameters (time and speed of the device, or number of elements used). A better blend may be obtained and maintained if the blend as a whole is slightly cohesive compared with a blend that is free flowing.

\section{Conclusion}

We can conclude that static mixers can be used for blending powders of different characteristics as well as the commonly used V-blenders or even as Turbula mixing devices which are used in pharmaceutical industry for very fine powders, especially if one component of the mixture is in a great surplus. Modelling of the fundamental mechanisms that can be applied in the design of a mixing process considering the particle motion can help define the final product and the process of mixing itself, especially when dealing with devices that are not commonly used for blending powdered and particulate matters. Alternative approach that deals with the application of chaos in mixing could lead to a more effective mixing with less energy requirement. The measuring flow rate of powders, as well as of mixtures, can help define the particles behaviour inside the mixture and therefore define the products quality. It can also predict the likelihood of the powder sticking to the surface and how the flow rate is affected by the composition of powder and processing conditions. It may seem easy with quartz sand and defined particle combinations, but these experiments should be done with real materials that contain many ingredients that have different particle properties, particularly in particle size which may lead to segregation or demixing after the mixing process, thus can seriously limiting the potential for creating ingredient mixes. The powder behaviour, especially powder flow in a blender, is a result of the combination of the equipment used and the material properties, which cannot be separated. 


\section{References}

Bauman I 2001 Solid-solid mixing with static mixers, Chem. Biochem. Eng. Q. 15(4): 159-165

Bauman I, Futač D 1996 Model of mixing solid materials, Kem. Ind. 45(9): 321.326

Gilbertson M A, Eames I 2003 The influence of particle size on the flow of fluidized powders, Powder Technol. 131: 197-205

Gyebis J, Katai F 1990 Determination and randomness in mixing of particulate solids, Chem. Eng. Sci. 45: 2843-2855

Gyebis J, Katai F 1990 Determination and randomness in mixing of particulate solids, Chem. Eng. Sci. 45: 2843-2855

Hersey J A 1982 Perfect powder mixtures, Powder Technol. 68: 287-296

Janssen R J M 2001 Structure and shear in a cohesive powder, Ph.D. thesis Technische Universiteit, Delft

Juliano P, B Muhunthan B, Barbosa-Ca'novas G V 2006 Flow and shear descriptors of preconsolidated food powders, J. Food Eng. 72: 157-166

Kaye B H 1997 Powder mixing, (London: Published by Chapman Chapman and Hall) Chapter 3: $107-131$

Lacey P M C 1943 Mixing of solid particles, Trans. Inst. Chem. Eng. 21: 53-59

Lakshman P, Cooney C L 2006 A review on the continuous blending of powders, Chem. Eng. Sci. 61: 720-742

Mort R. P., Riman E. R 1995 Determination of homogeneity scale in ordered and practically ordered Mixtures, Powder Technol. 82: 123-135

Muttio F J, Alexander A, Goodridge C L, Shen E, Shinbrot Z 2004 Solid Mixing Part A: Fundamentals of Solids Mixing. E L Paul, V A Atemio-Obeng, S M Kresta, (eds) The Handbook of Industrial Mixing Science and Practice (New York: Wiley)

Muzzio F J, Goodridge C L, Alexander A, Arratioa P, Youn H, Sudah O, Mergen G 2003 Sampling and characterization of pharmaceutical powders and granular blends, Int. J. Pharmaceutics 250: 51-64

Naranjan K, Smith D L O, Rielly C D, Limdley J A, Philips V R 1994 Mixing processes for agricultural and food materials. 5: Review of mixer types. J. Agricluture Res. 59: 145-161

Ottino J M, Khakhar D V 2000 Mixing and segregation of granular material. Annu. Rev. Fluid. Mech. 32: $55-64$

Paul W C, Metcalfe G, Liffman K 1998 How well do discrete element granular flow models capture essentials of mixing processes? Applied Mathematical Modelling 22: 995-1008

Pool K R, Taylor R F, Wall G P 1962 Mixing powders to a finale-scale homogeneity, studies of batchmixing, Trans. Inst. Chem. Eng. 42: 305-321

Prescott J K, Barnum R A 2000 On Powder Flowability, Pharmaceutical Technol. 10: 60-84

Railley C D, Smith D L O, Lindley J A, Niranjan K, Phillips V R 1994 Mixing processes for agricultural and food materials: Part 4, Assessment and Monitoring of Mixing Systems, J. Agric. Eng. Res. 59: $1-18$

Regner M, Ostergren K, Tragardh C 2006 Effects of geometry and flow rate on secondary flow and the mixing processes in static mixers-A numerical study, Chem. Eng. Sci. 61: 6133-6141

Ristow G H 2000 Mixing and segregation in rotating drums, Proc. Symposium on Segregation in Granular Flows, The Netherlands: Kluwer Academic publishers, 311-320

Tomas J 2004 Product design of cohesive powders-Mechanical properties, compression and flow behaviour, Chem. Eng. Technol. 27(6): 605-618

Turbula T2C Heavy-Duty Shaker-Mixer 2004 www.artisan-scientific.com/ 49853.asp accessed $12 \cdot 06 \cdot 2007$ 